\title{
Harvested Power Wireless Sensor Network Solution for Disaggregated Current Estimation in Large Buildings
}

\author{
Jose Pedro Amaro, Rui Cortesão, Member, IEEE, Jorge Landeck, \\ and Fernando J. T. E. Ferreira, Senior Member, IEEE
}

\begin{abstract}
In modern economies, large service buildings are responsible for an important part of the global electrical energy consumption. The implementation of energy saving strategies can benefit from disaggregated consumption monitoring. To tackle this problem, a number of technological solutions exist, being however, expensive in equipment, installation and maintenance. Because of its decentralized operation principle, wireless sensor networks (WSNs) are an important tool to implement disaggregated electrical energy monitoring. The development of a selfpowered, battery-free current sensor node for large WSNs may contribute to the implementation of monitoring solutions for large buildings. In this paper, a solution to monitor disaggregated consumption is presented, based on a contact-less power source for Zigbee nodes using a split-core toroidal coil current transformer (SCCT). The proposed device is able to power a battery-free wireless node estimating also the current drawn by the electrical load with a single SCCT. The SCCT is successfully applied to power a battery-free wireless device running a complex communication software stack. The proposed system is described through simulation as well as the experimental results.
\end{abstract}

Index Terms-Current, energy harvesting, estimation, IEEE 802.15 standards, wireless sensor networks, Zigbee.

\section{INTRODUCTION}

$\mathbf{T}$ HE wireless sensor networks (WSNs) [1]-[3] ability to collect and transfer information is the base of a number of new industrial projects. The most obvious energy source for a WSN node is the battery. Batteries have nevertheless a number of drawbacks that seriously limit the usage of WSN, and are in fact the main limitation to its widespread use. Low cost network implementation/operation will be achieved only if battery usage is substantially reduced or altogether eliminated. A simple calculation can be done: considering the deployment of a 500 node network, with $10 € / \mathrm{h}$ labor cost and $5 \mathrm{~min}$ for the actual deployment and commissioning process of each node, entails an approximate total cost of $417 €$. Considering the node cost determined in [4], the same 500 nodes cost less than

Manuscript received June 27, 2014; revised September 26, 2014; accepted November 11, 2014. Date of publication January 7, 2015; date of current version June 5, 2015. The associate editor coordinating the review process was Dr. Maciej Zawodniok.

J. P. Amaro and F. J. T. E. Ferreira are with the Department of Electrical Engineering, Instituto Superior de Engenharia de Coimbra, Coimbra 3030-271, Portugal, and also with the Institute of Systems and Robotics, University of Coimbra, Coimbra 3004-531, Portugal (e-mail: amaro@isec.pt).

R. Cortesão is with the Institute of Systems and Robotics, University of Coimbra, Coimbra 3004-531, Portugal.

J. Landeck is with Intelligent Sensing Anywhere, Porto 4200-355, Portugal

Color versions of one or more of the figures in this paper are available online at http://ieeexplore.iee.org.

Digital Object Identifier 10.1109/TIM.2014.2383052

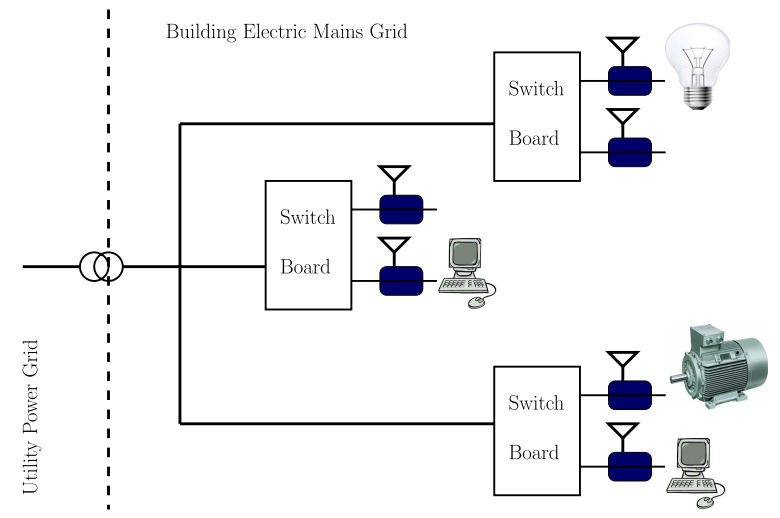

Fig. 1. Disaggregated mains current monitoring within a building electric installation using WSN

the actual deployment and commissioning process. The same reasoning can be done regarding maintenance costs that are mainly related to battery replacement in battery operated nodes. It is easy to imagine that large network prices grow with the number of nodes, generating prohibitive costs and unacceptable return of investment periods. Eliminating batteries is therefore an advantage in all environments, since monitoring a large number of electrical lines in large office buildings requires installation of a large number of wireless devices. Energy harvesting can be an important asset for a wireless network despite in-loco power availability. WSNs, whose nodes can easily be moved, may not require skilled workers for node placement and replacement, potentially lead to a significant cost reduction of network operation. In the context of a building electrical installation (as shown in Fig. 1), powering WSN nodes through the exclusive use of harvested energy is an important development. This development is relevant if a number of nodes are able to estimate the root mean square (rms) value of the current flowing in the power cables they scavenge energy from. Throughout the remaining of this document, the current flowing in power lines is addressed as mains current. Moreover, a WSN with the presented characteristics may be used to monitor user behavior, equipment efficiency, fault identification, and contribute to the implementation of the smart grid concept. In this paper, a current estimation device powered by a contact-less electromagnetic source is presented. A single split-core toroidal coil current transformer (SCCT) device scavenges energy from load power cables to power a WSN node. A IEEE 802.15.4/ZigBee communication protocol stack has been configured so that one such device runs without batteries. 
A number of solutions presented in the literature, may be identified as related to the work presented in this paper. In [5], a microcontroller controlled electromagnetic energy harvesting from a high-voltage transmission line is presented. This system is able to scavenge energy from line currents between 65 and $130 \mathrm{~A}$. A magnetic power generator and a voltage multiplier are used. In [6], a communication concept for inverter fed electric motors is presented. Powerline communication is used to send motor operational data. Power supply is obtained by inductive coupling from the remaining motor phases. The work presented in [7] reviews and compares available options for powering WSN nodes from large electric and magnetic fields which exist on highvoltage electrical installations, such as substations. In [8], a small electromagnetic energy harvesting device is proposed and several topologies for the magnetic coupler are tested. This energy harvesting device is used to charge a battery that can in turn power a wireless device. A free-standing inductive harvester for uses where a magnetic field induced by power cords is available, is investigated in [9]. In addition, in [10], a device is proposed that is able to obtain energy from the magnetic field induced by power cords. This device is able to scavenge energy from small currents. In [11], a medium-/high-voltage electric field harvester is presented and studied. A device that is able to harvest energy from the electric fields existing near high-voltage dc lines is presented in [12]. An ambient harvesting device with capacitor leakage awareness is investigated in [13]. A number of techniques for disaggregated end-use energy sensing for smart grids are surveyed in [14]. WSNs are an important tool to implement measurement systems [15]-[17]. In [18]-[21], energy harvesting devices are able to power IEEE 802.15.4/ZigBee compliant nodes. These contributions focus their analysis on the Zigbee data transfers and the sustainability of the system under regular communications. The IEEE 802.15.4/ZigBee protocol entails a number of nonregular communication action that are not addressed in these proposals. Our proposed device main contribution, is the ability to assess mains rms current value with a self-powered device that runs a complex WSN protocol. Our device scavenges energy from small rms mains currents and runs exclusively on capacitor stored energy. Moreover, this paper addresses Zigbee nonregular communications that entail large current drawing from the power source.

This paper is organized as follows. Section II describes the SCCT modeling process. A SPICE model describing the SCCT behavior is implemented and assessed using laboratory measurements. System power management is described in Section III. The proposed device operation is presented together with the storage management strategy. In Section IV, a description of relevant IEEE 802.15.4/ZigBee protocol operation is described. The power cable current estimation mechanism is discussed in Section V, and Section VI presents the conclusion.

\section{Parameter Identification and Modeling of the Magnetic Power Generator}

Voltage transformers and current sensing are examples of the widespread use of electromagnetic devices [5], [6].
TABLE I

MANUFACTURER VALUES FOR SCCT CHARACTERIZATION

\begin{tabular}{lllll}
$\begin{array}{l}\text { Core } \\
\text { area }\end{array}$ & $\begin{array}{l}\text { Magnetic } \\
\text { flux } \\
\text { length }\end{array}$ & $\begin{array}{l}\text { Secondary } \\
\text { turns }\end{array}$ & Core type & $\begin{array}{l}\text { Cross } \\
\text { section } \\
\text { area }\end{array}$ \\
\hline $5 \mathrm{~mm}^{2}$ & $5 \mathrm{~cm}$ & 3000 & $\begin{array}{l}\text { Ferrite } \\
\text { (Ui: } 7000\end{array}$ & $50 \mathrm{~mm}^{2}$ \\
& & & $\begin{array}{l}\text { Bs:4300 } \\
\mathrm{mT})\end{array}$ & \\
& & & &
\end{tabular}

In this paper, a magnetic power generator is used to build a contactless energy harvesting system for a wireless module. An SCCT is used to generate a current from the induced electromotive force. The toroidal coil scavenges energy from wires connecting electric charges (e.g., three-phase induction motor power cables) powering a wireless sensor node.

Ampere's law allows the determination of the current generated by the magnetic generator, given by

$$
\oint H . d l=i_{p}-n i_{s}
$$

where $H$ is the magnetic field density, $i_{p}$ is the primary current (in this case mains current), $n$ is the number of turns in the transformer secondary circuit, and $i_{s}$ is the current generated in the secondary coil.

Applying Faraday's law, $V=n d \phi / d t=i_{S} R$, (1) can be written as

$$
I_{s} R=(n \mu A / \ell) d / d t\left(i_{p} / n-i_{s}\right) .
$$

Secondary coil current for a load $R$ can therefore be obtained for node energy supply.

In this paper, a SPICE circuit simulator is used and the SCCT is modeled by a pair of mutually coupled inductors. Modeling the SCCT requires however, circuit parameters that are not provided by the SCCT manufacturer. Moreover, SCCT is commonly used in current sensing applications. Their datasheets are for different uses that the one pursued in this paper. In addition, secondary inductor values are missing from manufacturer datasheet, whereas the use of SPICE mutual induction model requires primary and secondary induction knowledge. These values are obtained through an experimental setup to determine a first estimate coil values followed by simulation for fine tuning. The obtained SPICE model is then applied in a simulated environment to subsequent system design and implementation. The known values prior to experimental setup measurements are shown in Table I. The first estimate for primary and secondary inductor values is obtained analytically from values delivered by manufacturer and read from the experimental setup. The coil is placed around a power line with a fixed current thus generating an output voltage. Considering that transformer universal electromotive force equation for a single-frequency circuit can be expressed as

$$
E_{\mathrm{rms}}=4.44 f n \phi
$$

where $E_{\text {rms }}$ is the voltage generated at the SCCT output, $f$ is the circuit operating single frequency, $\phi$ is the generated magnetic flux, and $n$ is the number of secondary turns, the open-circuit SCCT output voltage is measured thus allowing 

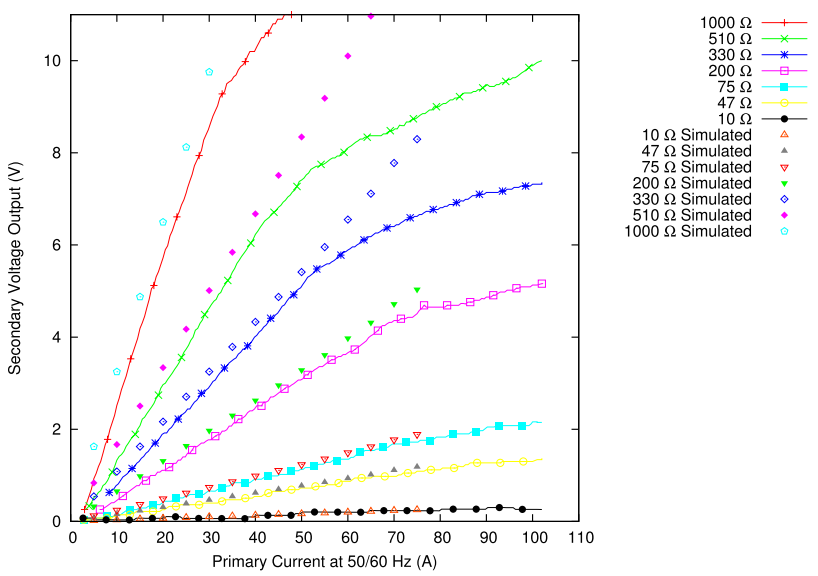

Fig. 2. Datasheet split-core toroidal coil $I-V$ curves versus simulated curves for coupled inductor model.

TABLE II

Computed Values for Split-Core Toroidal Core Spice Model

\begin{tabular}{llll}
$\begin{array}{l}\text { First } \\
\text { estimate } \\
\text { for primary } \\
\text { induction } \\
\text { value }\end{array}$ & $\begin{array}{l}\text { First } \\
\text { estimate for } \\
\text { secondary } \\
\text { induction } \\
\text { value }\end{array}$ & $\begin{array}{l}\text { Model value } \\
\text { for primary } \\
\text { inductance }\end{array}$ & $\begin{array}{l}\text { Model } \\
\text { value for } \\
\text { secondary } \\
\text { coil } \\
\text { inductance }\end{array}$ \\
\hline $5 \mu \mathrm{H}$ & $66 \mathrm{H}$ & $5 \mu \mathrm{H}$ & $45 \mathrm{H}$
\end{tabular}

the determination of the magnetic flux. Magnetic flux is the link between primary current $i$ and the open-circuit secondary voltage. This link may be expressed by the $n \phi=L i$ relation that with (3) allows the determination of a first estimate of the primary coil inductance $L$.

For the secondary coil induction (4) and (5) are used

$$
L=\frac{n^{2}}{\mathcal{R}}
$$

where $\mathcal{R}$ is the magnetic reluctance and $L$ is the secondary coil inductance. Coil reluctance is obtained from (5) relating its value with toroid geometry and core materials

$$
\mathcal{R}=\frac{l}{\mu_{0} \mu_{r} A}
$$

where $l$ is the path length for the magnetic flux, $\mu_{0}$ is the magnetic permeability of the air, $\mu_{r}$ is the relative magnetic permeability of ferrite, and $A$ is the cross-sectional area of the secondary core. Fig. 2 shows the SCCT output voltage for a number of resistive loads as announced by the manufacturer and the simulated values obtained from the developed model. It can be inferred that the implemented SCCT SPICE model is valid for mains currents up to $30 \mathrm{~A}$. For higher current values, the saturation effect leads to a nonlinear behavior and the model is no longer valid. For current values up to $30 \mathrm{~A}$ the saturation effect is considered negligible.

Fine tuning and analytical estimates of primary and secondary induction SCCT values are shown in Table II.

\section{Proposed Solution for a Harvested Powered IEEE 802.15.4/ZIGBEE DEVICE}

Powering a WSN node directly from the SCCT is not a feasible solution. A conversion circuit is therefore built.

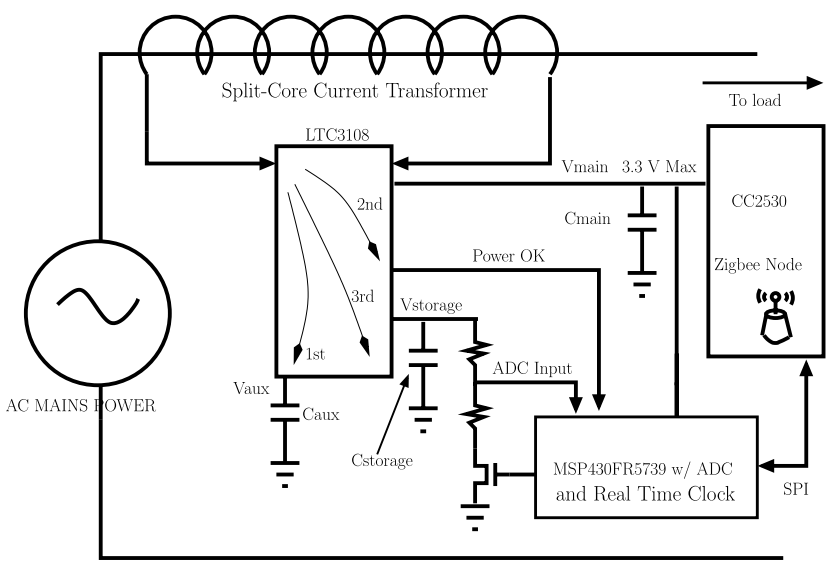

Fig. 3. Energy harvester architecture illustrating capacitor notation. $C_{\text {storage }}$ identifies a capacitor connected to the LTC3108 line whose voltage is identified as $V_{\text {storage. An identical notation is applied to } C_{\text {main }} \text { and } C_{\text {aux }}}$ capacitors.

The implemented ac/dc conversion circuit is based on the LTC3108 [22] from linear technology. The proposed system uses LTC3108 as a bootstrap charge manager for the device energy storage capacitors. The energy thus stored is thereafter used to power a radio-frequency (RF) module as well as a low-power device manager. Fig. 3 shows the proposed system architecture. The RF module is implemented with a CC2530 [23] Zigbee device, while the system power manager uses an MSP430FR5739 [24] microcontroller both from Texas Instruments. The proposed system is a self-powered monitoring device that may be used to estimate the average rms current values of mains power cable installations. It is able to estimate load current consumption while being powered through a single contactless electromagnetic power source using only one SCCT. The device acts as a wireless network node sending collected measurements through the network.

The system operation may be described by three distinct phases: charging, measurement, and energy transfer for communication. In the charging phase, the device scavenges energy from load power cables until the device manager operation is possible. The manager operation allows mains current measurements and surplus energy use on the RF module. Capacitors $C_{\text {aux }}, C_{\text {main }}$, and $C_{\text {storage }}$ behavior can be used to characterize the charging phase. The first operation charges $C_{\text {aux }}$ up to $2.2 \mathrm{~V}$, where the capacitor $C_{\text {main }}$ starts charging. Energy stored at $C_{\text {aux }}$ is used to power LTC3108 internal circuitry and $C_{\text {main }}$ may be used to power an external load. $C_{\text {main }}$ voltage is hardware configured and for this paper is set to $3.3 \mathrm{~V}$. Once $C_{\text {main }}$ is fully charged, the excess energy is used to charge $C_{\text {storage }}$ that in turn may be used to recharge $C_{\text {main }}$ if harvested energy fails. $C_{\text {storage }}$ may charge up to $5.25 \mathrm{~V}$, grounding all excess harvested energy. Fig. 4 shows the implemented solution. A modular device is proposed thus allowing diverse implementations architectures (e.g., change the RF module and operate with different capacitor sets).

\section{A. Capacitor Choice Issues}

Capacitor choices have critical influence on system behavior. Both capacitor value and leakage current are relevant 


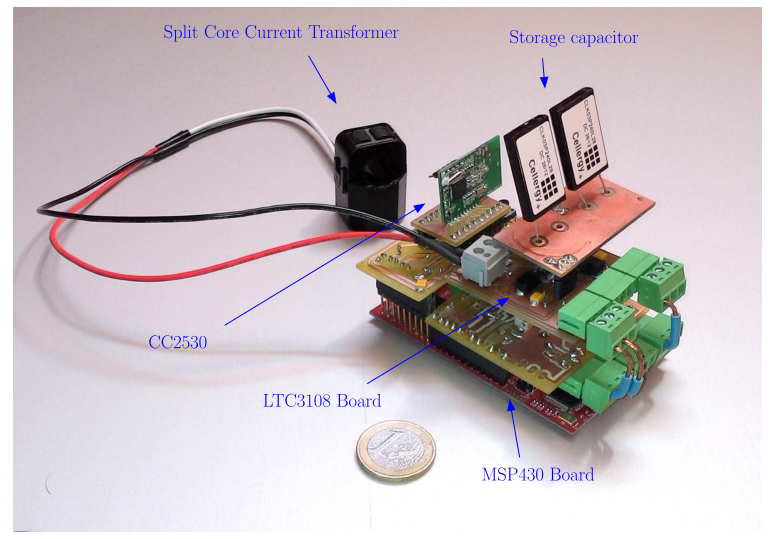

Fig. 4. Photo of implemented device showing modular design.

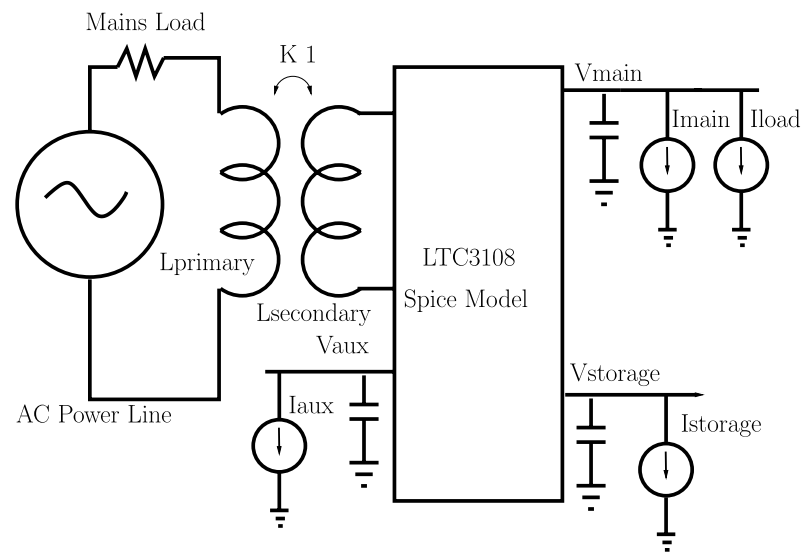

Fig. 5. SPICE simulation model of the proposed system.

design considerations with critical influence on charge times. Moreover, in the absence of mains current, capacitor leakage current is relevant for system lifetime even without RF activity. The choice of capacitor devices is therefore limited to those with low-leakage characteristics. Leakage capacitor current is outside the scope of this paper and is addressed in [13] and [25]. Two different capacitor sets have been tested. One set has been built with Vishay aluminum capacitors [26], as advised in LTC3108 datasheet. This set is built with two $330 \mu \mathrm{F}$ (for both $C_{\text {main }}$ and $C_{\text {storage }}$ ) capacitors. Another set has been implemented with larger capacitance values that are obtained from Cellergy [27]. These capacitors present the desired characteristics of high-capacity and low self-discharge current. A $240-\mathrm{mF}$ capacitor for $V_{\text {main }}$ and a $120-\mathrm{mF}$ capacitor for $V_{\text {storage }}$ have been implemented. The capacitance choice value is justified in subsequent sections.

\section{B. System Modeling}

LTC3108 linear technology provided SPICE model and the model presented in Section II are used to develop and study the proposed solution. Fig. 5 illustrates the implemented circuit SPICE model, where the SCCT is used as a secondary transformer winding connected to the LTC3108 input. The proposed model accounts for capacitor leakage currents using current sources, as shown in Fig. 5. Current sources are

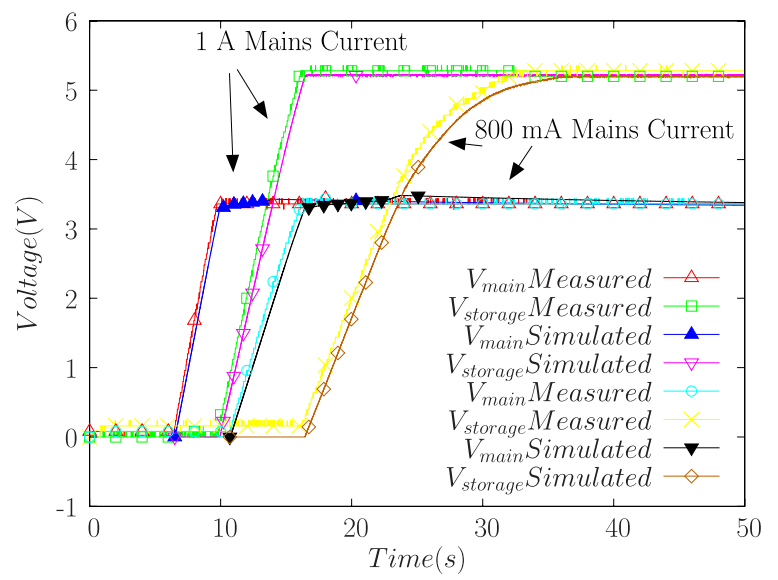

Fig. 6. Simulated and measured LTC3108 operation for $1 \mathrm{~A}$ and $800 \mathrm{~mA}$ mains current using $330 \mu \mathrm{F}$ capacitors.

added to the model so that capacitor leakage current effects are accounted for. In Fig. 6, measured and simulated device behavior are illustrated for $1 \mathrm{~A}$ and $800 \mathrm{~mA}$ mains current and $330 \mu \mathrm{F}$ capacitors as advised in LTC3108 datasheet. The model correctly predicts capacitor charging operation when compared with real measurements. Table III shows measured and simulated device charge times for mains current ranging from $500 \mathrm{~mA}$ to $1.2 \mathrm{~A}$ for both capacitor sets. Charge time is measured for capacitor full charge thus representing a worst case scenario for system operation. The simulated charge times for large cellergy capacitors are presented for capacitor charge starting at $4 \mathrm{~V}$. The following sections show the relevance of this option thus preventing large SPICE simulations that are difficult to perform using regular computational resources.

\section{Understanding Zigbee Operation}

IEEE 802.15.4/ZigBee is a well-specified, studied, and described standard. Moreover, a number of protocol stack implementations are freely distributed [28]-[30], making it a very useful tool for test and verification of WSN with an added characteristic of small time to market implementation. To the purpose of this paper, the choice of this complex protocol is justified by the fact that if the system is able to operate using IEEE 802.15.4/ZigBee, it will also operate with a different protocol with a simpler implementation. The system is programmed using Texas Instruments Zigbee protocol stack (Z-Stack [28]). Zigbee operation and concomitant power consumption is not exclusively dependent on programming. Node behavior and drawn current are highly dependent on environmental conditions (e.g., RF propagation condition) and network operation [31]. Moreover, unlike battery powered nodes, a device running exclusively on capacitor charge may easily run into power failure. This event occurs if the microprocessor power line lowers under its operational limits. In [23], this voltage limit is announced to be $1.8 \mathrm{~V}$.

Zigbee operation requires that the Join procedure [32], [33] is executed once by the nodes. Prior to joining the network each node must execute a network scan, thus choosing a suitable parent using design parameters. Network scans [34], [35] 
TABLE III

Simulated and Measured Contact-Less Energy Harvesting Device. Charging Time for Different Mains Current and Capacitor Values. Two Capacitor Sets Charge Times Are Shown. One Set Is Implemented With a $2.2 \mu$ F $C_{\text {aux }}$ Capacitor AND Two $330 \mu \mathrm{F}$ (FOR Both $C_{\text {main }}$ AND $C_{\text {storage }}$ ). The SeCOND Set Is Built With a $2.2 \mu \mathrm{F} C_{\text {aux }}$ A $240 \mathrm{mF}$ CAPACITOR FOR $C_{\text {main }}$, AND A $120 \mathrm{mF}$ CAPACITOR FOR $C_{\text {storage }}$

\begin{tabular}{|c|c|c|c|c|c|c|}
\hline \multicolumn{2}{|c|}{ Mains Current } & $V_{\operatorname{main}}\left(3.3 \mathrm{~V}^{*}\right)$ & $V_{\text {storage }}\left(5.2 \mathrm{~V}^{*}\right)$ & \multirow{4}{*}{$\begin{array}{c}V_{\operatorname{main}}\left(3.3 \mathrm{~V}^{*}\right) \\
240 \mathrm{mF} \text { Capacitor } \\
C_{\text {main }} \text { full charge }\end{array}$} & $V_{\text {storage }}\left(5.2 \mathrm{~V}^{*}\right)$ & $V_{\text {storage }}\left(5.2 \mathrm{~V}^{*}\right)$ \\
\hline & & \multicolumn{2}{|c|}{ First capacitor set } & & Second cap & itor set $\longrightarrow$ \\
\hline & & \multicolumn{2}{|c|}{$330 \mathrm{uF}$ Capacitor } & & $120 \mathrm{mF}$ Capacitor & $120 \mathrm{mF}$ Capacitor \\
\hline & & $C_{\text {main }}$ full charge & $C_{\text {storage }}$ full charge & & $C_{\text {storage }}$ full charge & $C_{\text {storage }}$ charge from $4 \mathrm{~V}$ to $5.27 \mathrm{~V}$ \\
\hline \multirow[t]{2}{*}{$400 \mathrm{~mA}$} & Measured & N" & 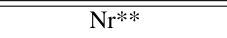 & 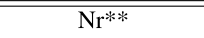 & $\begin{array}{ll}\mathrm{Nr} * * \\
\end{array}$ & $\begin{array}{ll}\mathrm{Nr} * * \\
\end{array}$ \\
\hline & Simulated & $\mathrm{Nr} * *$ & $\mathrm{Nr}^{* *}$ & $\mathrm{Nr}^{* *}$ & $\mathrm{Nr} * *$ & $\mathrm{Nr} * *$ \\
\hline \multirow[t]{2}{*}{$500 \mathrm{~mA}$} & Measured & $15.2 \mathrm{~s}$ & $42 \mathrm{~s}(3.12 \mathrm{~V})$ & $17 \mathrm{~h}: 30 \mathrm{~m}: 20 \mathrm{~s}$ & $(0.34 \mathrm{~V})$ & $(0.34 \mathrm{~V})$ \\
\hline & Simulated & $17 \mathrm{~s}$ & $52 \mathrm{~s}(3.2 \mathrm{~V})$ & - & - & 14h:00m:00s \\
\hline \multirow[t]{2}{*}{$600 \mathrm{~mA}$} & Measured & $7.2 \mathrm{~s}$ & $32 \mathrm{~s}(3.76 \mathrm{~V})$ & $07 \mathrm{~h}: 02 \mathrm{~m}: 35 \mathrm{~s}$ & 17h:00m:00s $(4.3 \mathrm{~V})$ & 01h:20m:04s (4.0V - 4.3V) \\
\hline & Simulated & $9.1 \mathrm{~s}$ & $36 \mathrm{~s}(3.8 \mathrm{~V})$ & - & - & - \\
\hline \multirow[t]{2}{*}{$700 \mathrm{~mA}$} & Measured & $9.0 \mathrm{~s}$ & $23 \mathrm{~s}(4.16 \mathrm{~V})$ & 05h:08m:40s & $10 \mathrm{~h}: 37 \mathrm{~m}: 00 \mathrm{~s}(4.7 \mathrm{~V})$ & $01 \mathrm{~h}: 10 \mathrm{~m}: 20 \mathrm{~s}(4.0-4.7 \mathrm{~V})$ \\
\hline & Simulated & $8.9 \mathrm{~s}$ & $29 \mathrm{~s}(4.9 \mathrm{~V})$ & - & - & - \\
\hline \multirow[t]{2}{*}{$800 \mathrm{~mA}$} & Measured & $4.8 \mathrm{~s}$ & $17 \mathrm{~s}$ & 03h:07m:35s & $05 \mathrm{~h}: 45 \mathrm{~m}: 59 \mathrm{~s}$ & 01h:03m:20s \\
\hline & Simulated & $5.6 \mathrm{~s}$ & $22.9 \mathrm{~s}$ & - & - & - \\
\hline \multirow[t]{2}{*}{$900 \mathrm{~mA}$} & Measured & $3.8 \mathrm{~s}$ & $19.2 \mathrm{~s}$ & $01 \mathrm{~h}: 36 \mathrm{~m}: 20 \mathrm{~s}$ & 03h:19m:40s & 00h:41m:20s \\
\hline & Simulated & $4.9 \mathrm{~s}$ & $20.8 \mathrm{~s}$ & - & - & $00 \mathrm{~h}: 43 \mathrm{~m}: 12 \mathrm{~s}$ \\
\hline \multirow[t]{2}{*}{$1000 \mathrm{~mA}$} & Measured & $3.0 \mathrm{~s}$ & $13.6 \mathrm{~s}$ & 01h:30m:00s & 03h:10m:00s & $00 \mathrm{~h}: 35 \mathrm{~m}: 40 \mathrm{~s}$ \\
\hline & Simulated & $4.2 \mathrm{~s}$ & $14.4 \mathrm{~s}$ & - & - & $00 \mathrm{~h}: 32 \mathrm{~m}: 56 \mathrm{~s}$ \\
\hline \multirow[t]{2}{*}{$1200 \mathrm{~mA}$} & Measured & $2.8 \mathrm{~s}$ & $10.1 \mathrm{~s}$ & 01h:09m:20s & $02 \mathrm{~h}: 18 \mathrm{~m}: 40 \mathrm{~s}$ & $00 \mathrm{~h}: 24 \mathrm{~m}: 00 \mathrm{~s}$ \\
\hline & Simulated & $3.23 \mathrm{~s}$ & $11.78 \mathrm{~s}$ & - & - & $00 \mathrm{~h}: 23 \mathrm{~m}: 21 \mathrm{~s}$ \\
\hline
\end{tabular}

* Maximum voltage adjusted for this capacitor

** Neither capacitor reached maximum voltage

Active Scan and Network Join

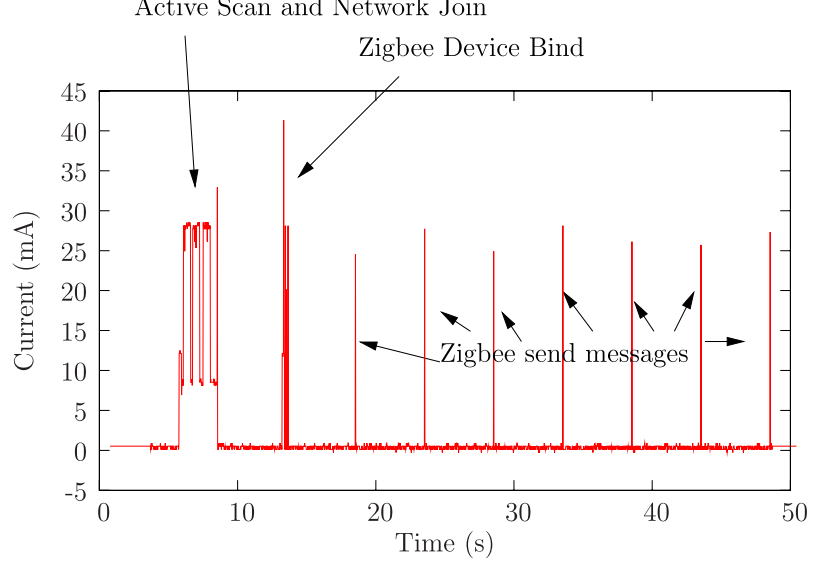

Fig. 7. Current absorbed by the CC2530 ZED operating a Zigbee compliant stack. Obtained with our setup, measuring current consumption of a ZED in a simple two node network. The setup measures the voltage drop on a $1 \Omega$ resistor in series with the CC2530 microcontroller voltage line.

consist of a sequence of beacon requests to determine the existence of network coordinators or routers using different radio channels. This operation entails a prolonged turn on time for the RF module. In addition, the Zigbee specification defines application objects to implement desired functionalities. Two nodes with linked functionalities share common application object structures. Data transfers are possible by encapsulating these data structures into the transmitted frames. A Zigbee end device (ZED) [29] node full operation with active scan/join, bind [28] and data transfer actions is shown in Fig. 7. Fig. 7 is obtained by measuring current consumption of a ZED in a simple testbed with one Zigbee coordinator [29] and applying the procedure described in [36]. As illustrated, data transfers lead to less energy consumption than the

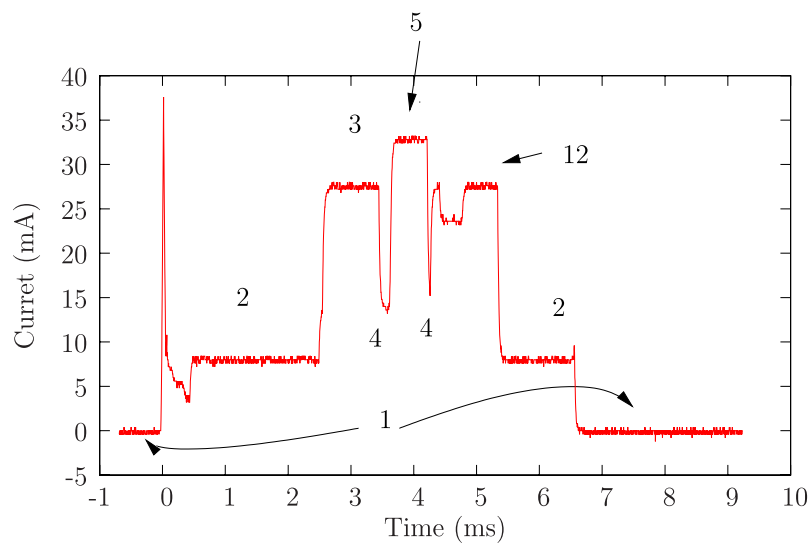

Fig. 8. Drawn current during data requests with MAC layer acknowledgement. Obtained with our setup, measuring current consumption of a ZED in a simple two node network. The setup measures the voltage drop on a $1 \Omega$ resistor in series with the CC2530 microcontroller voltage line.

join/active scan procedures. Moreover, within the shown 50-s test, energy consumption of joining the network using an active scan procedure is the system main energy constraint. Fig. 8 shows drawn current during an example data transfer. This figure shows the current required for a frequent action executed in low-power ZED, where the node polls its coordinator data and waits for requested data. One such data transfer entails a sequence of actions [36] that are easily identified by the numbering. Time slot 1 corresponds to a current related to the CC2530 power mode, in which only one timer and a low-frequency oscillator are running. The microcontroller wakeup time interval 2 is related with the startup sequence when the microcontroller enables its $32 \mathrm{MHz}$ oscillator. A CSMA/CA [37], [38] algorithm is executed in time slot 3. 


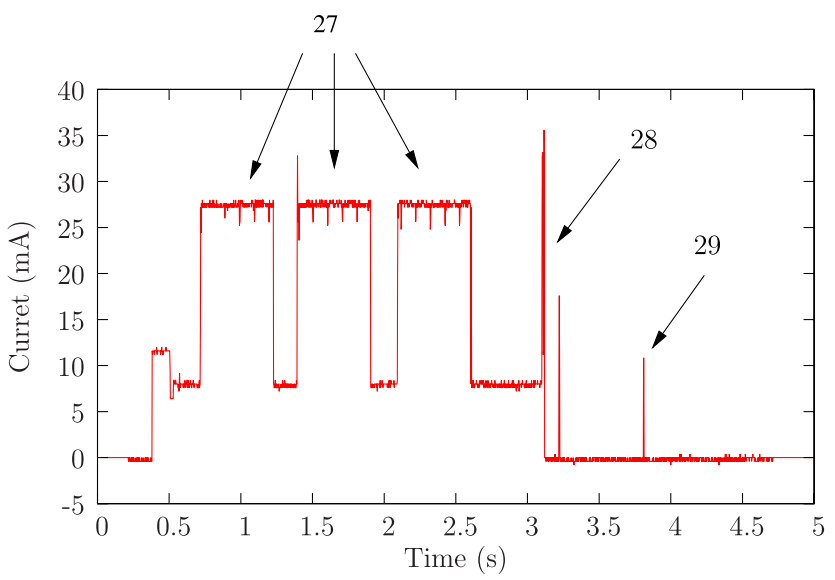

Fig. 9. Absorbed current for active scan and association with a Zigbee device. Obtained with our setup, measuring current consumption of a ZED in a simple two node network. The setup measures the voltage drop on a $1 \Omega$ resistor in series with the CC2530 microcontroller voltage line.

In this slot, the node repeatedly executes a clear channel assessment (CCA) with a backoff mechanism which presents variable time length. Prior to data transmission, nodes execute a radio wave carrier sense to determine if other nodes are transmitting. The first CCA is executed after a random backoff period. If the radio channel is busy a new backoff period is measured until a new CCA is executed. Time slots 4 have fixed length and are related with radio module switching between receiving and transmission modes. Data are transmitted in time slot 5 whose length is related to the fixed number of bytes this specific command sends. Time slot 12 is also fixed time and relates to sending or receiving Media Access Control (MAC) level acknowledgment without CSMA/CA mechanism. In Fig. 9, active scan and network join procedures are shown. In nonbeacon Zigbee networks, a node wishing to join the network first searches for the best parent. The best parent is selected by issuing a beacon request using a command frame and listening for answers. This action may be repeated for several radio channels. A node must therefore remain in active Rx mode for a long period of time as shown by the time interval 27 in Fig. 9. The time slot 28 shows the required time length to execute a join operation as previously described. Table IV summarizes current consumption required for Zigbee data exchanges.

\section{Estimating Mains CurRent}

Portable or otherwise independent devices are commonly powered by batteries that provide a stable voltage level. The proposed battery-less device also provides a stable voltage level, relying exclusively on its capacitor stored energy to power an RF module and two microcontrollers. Unlike a battery operated device, once uncontrolled currents are drawn from the capacitor, voltage may drop, failing to power the microcontrollers. The proposed system operation should meet these energy constraints preventing $C_{\text {main }}$ from discharging bellow minimum voltage required for microcontroller operation. After $C_{\text {storage }}$ full charge, our system is able to estimate mains current average value by promoting capacitor discharge and estimating the slope of the recharging voltage. The system operation is characterized by two modes: 1) an
TABLE IV

CURRENT CONSUMPTION REQUIRED FOR THE ZigBeE DATA EXCHANGES ILLUSTRATED IN FIgS. 8 AND 9

\begin{tabular}{|c|c|c|}
\hline Slot & Time Length & Current \\
\hline 1 & $\begin{array}{l}\text { Variable. Time between two con- } \\
\text { secutive transmission }\end{array}$ & $1 \mu A$ \\
\hline 2 & $\begin{array}{l}\text { Application dependent data pro- } \\
\text { cessing. Processor runs with the } \\
32 \mathrm{Mhz} \text { oscillator, for this example } \\
2.5 \mathrm{~ms}\end{array}$ & $0.76 \mathrm{~mA}$ \\
\hline 3 & $\begin{array}{l}\text { CSMA/CA algorithm. Variable } \\
\text { length. } 1.4 \mathrm{~ms} \text { for this example. }\end{array}$ & $27 \mathrm{~mA}$ \\
\hline 4 & $\begin{array}{l}\text { Switching } \mathrm{Tx} / \mathrm{Rx} \text { or } \mathrm{Rx} / \mathrm{Tx} \text { radio } \\
\text { module. Fixed } 190 \mu \mathrm{s}\end{array}$ & $14 \mathrm{~mA}$ \\
\hline 5 & $\begin{array}{l}\text { Data transmission. Fixed number } \\
\text { of bytes, } 900 \mu \mathrm{s}\end{array}$ & $33 \mathrm{~mA}$ \\
\hline 12 & $\begin{array}{l}\text { MAC Layer Acknowledgement } \\
970 \mu \mathrm{s}\end{array}$ & $33 \mathrm{~mA}$ \\
\hline 27 & $\begin{array}{l}\text { Three Channel Scan procedures } \\
\text { with aprox. } 1 \mathrm{~s} \text { each. }\end{array}$ & $27 \mathrm{~mA}$ \\
\hline 28 & $\begin{array}{l}\text { Join procedure. Variable length en- } \\
\text { tailing a number of data transfers. }\end{array}$ & Variable \\
\hline 29 & Data request as illustrated in Fig. 8 & Variable \\
\hline
\end{tabular}

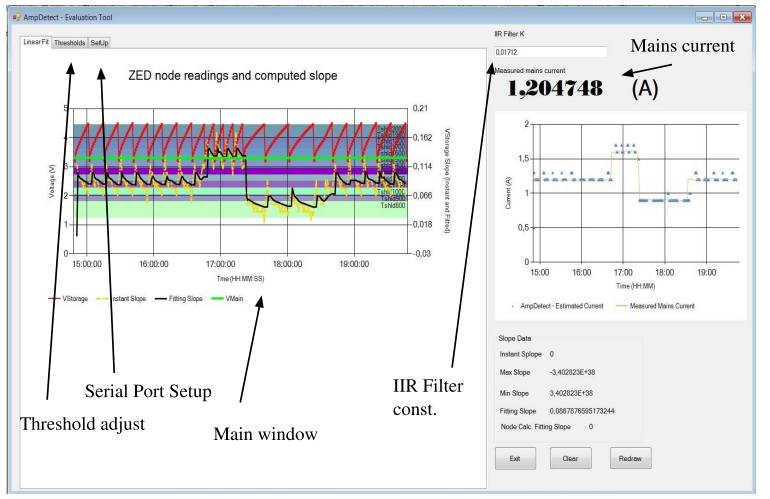

Fig. 10. Visual Studio/C\# implemented GUI. The GUI collects data from the estimation device Zigbee network and the implemented ammeter USB cable.

active mode where controlled capacitor discharge is executed and 2) both microcontrollers are active. A low-power mode where both processors are sleeping, and only the MSP430 real time clock is continuously operating with Analog to Digital Converter (ADC) periodic reads. In this case, the system periodically executes IEEE 802.15.4/ZigBee data transfers after measuring $V_{\text {storage }}$ level. The instant $V_{\text {storage }}$ slope between two consecutive samples is computed and accumulated by a curve fitting algorithm thus obtaining an average slope. The average value is compared against a lookup table to estimate mains current.

Mains current is measured and compared with the obtained estimate. The measured results have been obtained by our implementation of a digital ammeter. The ammeter has been built with a well-known implementation of an SCCT connected to a microcontroller through a voltage divider. The microcontroller implements an infinite impulse response (IIR) filter to remove the dc component and computes the rms value of the current, as described in [39] and [40]. This measurement device is implemented with a second MSP430FR5739 board that is connected to a graphical user interface (GUI) framework by an universal serial bus (USB). Moreover, mains current measurements are corroborated using a hand-held 


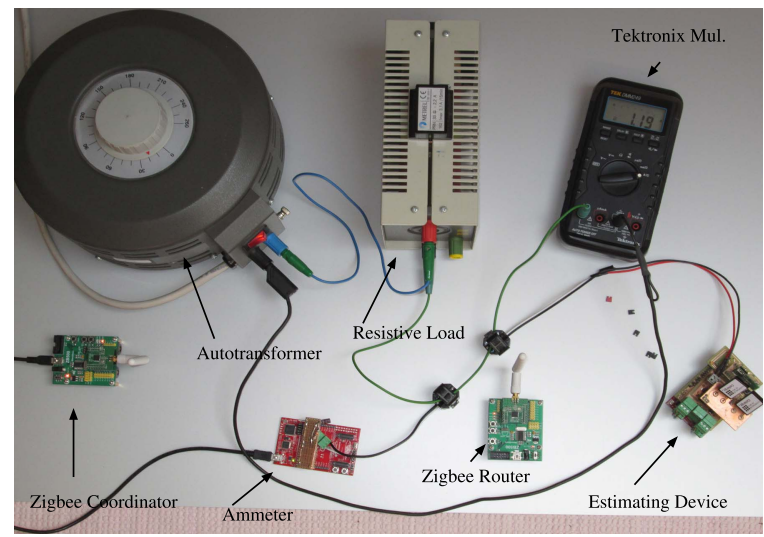

Fig. 11. Implemented test setup with the Zigbee nodes, the estimating and ammeter devices, the charge cables, the HSN-0303 autotransformer, the Tektronix multimeter, and the resistive load.

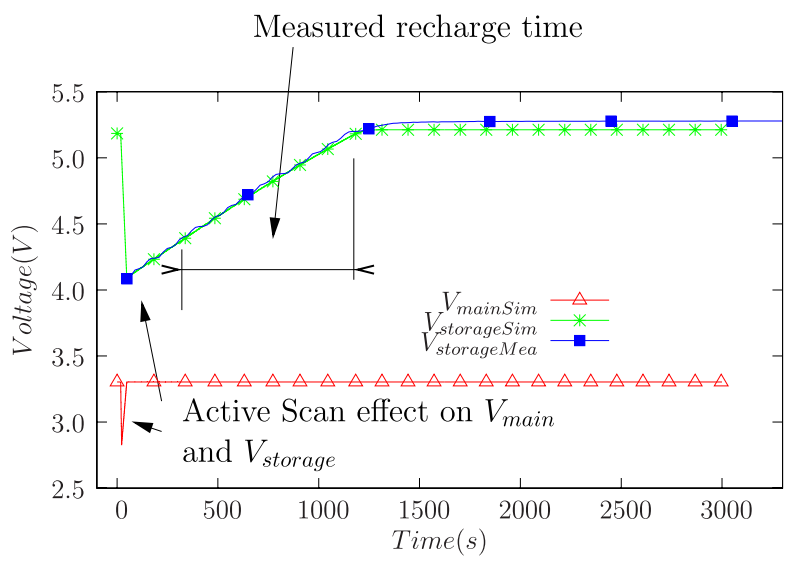

Fig. 12. $V_{\text {storage }}$ and $V_{\text {main }}$ voltage levels for active scan, association, and data communication for ZED over $1 \mathrm{~h}$. $V_{\text {storageSim }}$ and $V_{\text {mainSim }}$ are simulated values. $V_{\text {storageMea }}$ presents measured results.

Tektronix DMM249 multimeter that is used for mains current visual verification as well as to adjust the IIR filter characteristic constant. The implemented framework with the GUI is shown in Fig. 10. The framework receives data from the Zigbee network sink node as well as from our ammeter. Fig. 11 shows the implemented test setup showing the Zigbee nodes, the estimating and the measurement devices. The current source is a Metrel 3.38 KW HSN-0303 variable autotransformer connected to a resistive load. Fig. 12 shows the described mechanism where a $C_{\text {main }}$ controlled discharge is executed. In this scenario, the $C_{\text {storage }}$ capacitor energy is used for immediate $C_{\text {main }}$ recharge, and $C_{\text {storage }}$ starts recharging from harvested energy. Fig. 12 shows both measured and simulated $V_{\text {storage }}$ variation for 1-A mains current. The system power manager is able to estimate mains current by analyzing the slope of the $V_{\text {storage }}$ recharge. The estimation process identifies a discrete value for $I_{\text {mains }}$ that is close to its average value. $C_{\text {main }}$ controlled discharge is achieved by executing an active scan procedure. The current drawn by this procedure is software controlled and may therefore be used without capacitor overdischarging. In our system, CC2530 minimum operation voltage is $1.8 \mathrm{~V}$ and the MSP430 operates with line voltages as low as $2.0 \mathrm{~V}$. The active scan effect in $V_{\text {main }}$
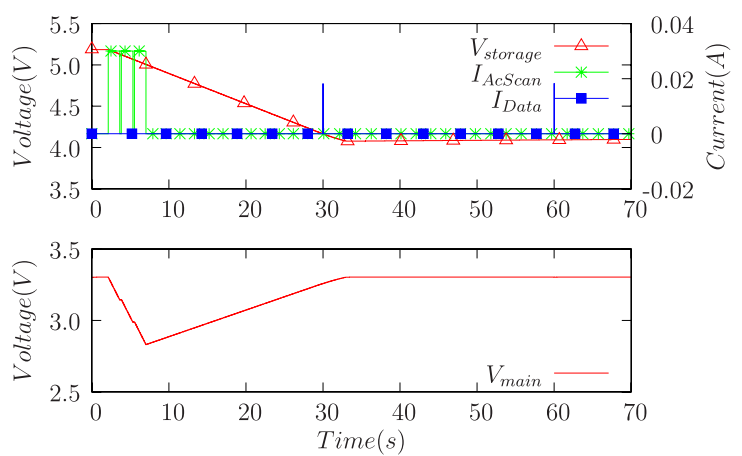

Fig. 13. Active scan and association Zigbee device related drawn current and node voltage variation. $V_{\text {storage }}$ is the simulated $C_{\text {storage }}$ voltage, $I_{\text {Data }}$ is the simulated current drawn by a data request, and $I_{\text {AcScan }}$ is the simulated drawn current from an active scan procedure.

is shown in Fig. 13, which corresponds to a zoom of the first $70 \mathrm{~s}$ of Fig. 12. The current consumption of the active scan procedure is illustrated by $I_{\mathrm{AcScan}}$ and data transfers are illustrated by current $I_{\text {Data }}$. Fig. 13 shows that the active scan procedure is an important energy consumer when compared with data transfer actions, which occur around 30 and $60 \mathrm{~s}$. It can be inferred that $I_{\text {Data }}$ influence is small on both $C_{\text {main }}$ and $C_{\text {storage }}$ charge values. The chosen $C_{\text {main }}$ capacitance of $240 \mathrm{mF}$ is justified by its ability to sustain out-of-the-box Zstack operation of three active scan procedures. The same capacitors are serially connected to form a single $C_{\text {storage }}$ device due to higher operation voltage. This sequence of operations is controlled by the MSP430 system power manager. Fig. 14 shows the system work-flow diagram. The MSP430 ADC is used to determine the lowest voltage level reached by $V_{\text {storage. }}$ Once $V_{\text {storage }}$ reaches $V_{\text {low }}$ (which is set to $3.0 \mathrm{~V}$ ), the recharge time is started for the mains current estimation process. When the recharge process starts, all microcontrollers are halted and the system is placed in low-power mode. The activity is related to ADC periodic $V_{\text {storage }}$ measurements to determine charge state. Periodic $V_{\text {storage }}$ readings are obtained and transferred to the Zigbee sink node with a 1-min interval. A $16 \mathrm{~B}$ data structure is transferred by the device. Within this structure $2 \mathrm{~B}$ store the $V_{\text {storage }}$ value, $4 \mathrm{~B}$ are used for the universal time coordinated, $2 \mathrm{~B}$ store the sender Zigbee short address and $2 \mathrm{~B}$ transfer the $V_{\text {main }}$ voltage. The remaining $6 \mathrm{~B}$, though transferred, do not carry useful data and may the used for the system future development, e.g., transfer temperature and/or humidity data. A timeout is used to prevent the system from indefinitely waiting for the $V_{\text {storage }}$ to reach $4.3 \mathrm{~V}$. The system does not provide mains current estimations in this case, indicating an under $800 \mathrm{~mA}$ value.

Figs. 15 and 16 show $V_{\text {main }}$ and $V_{\text {storage }}$ full charge time also indicating voltage bound for the algorithm application. It can be inferred that different mains currents entail different $V_{\text {storage }}$ voltage rise slopes. The instantaneous rise between two consecutive $V_{\text {storage }}$ readings present scattered values which are not adequate for direct mains current estimation. Therefore, a least squares method [41]-[43] is applied. For a given set of instantaneous $V_{\text {storage }}$ slopes $\left\{\left(t_{0}, \mathrm{Vst}_{\text {InstSlope }}(0)\right), \ldots,\left(t_{N}, \mathrm{~V}\right.\right.$ st $\left.\left._{\text {InstSlope }}(N)\right)\right\} \quad$ with $N$ samples, the best linear fitting is given by $y=M x+B$ 


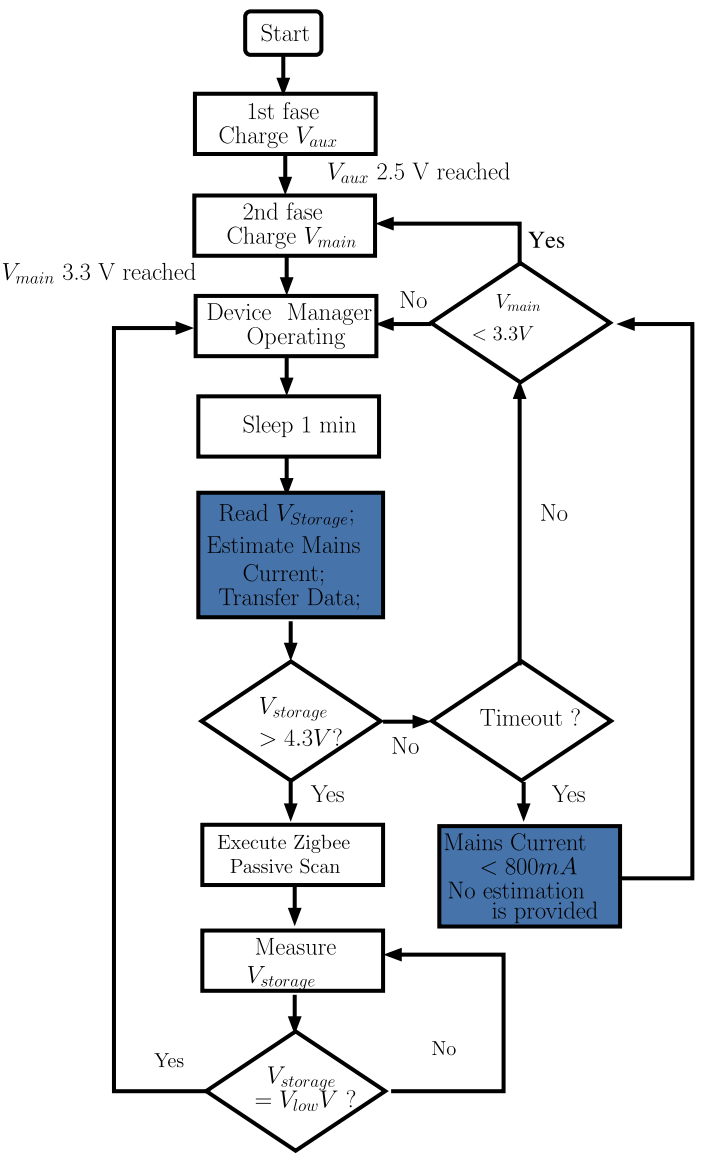

Fig. 14. System power manager work flow diagram for $C_{\text {storage }}$ charge/discharge mechanism, mains current estimation, and data transfer.

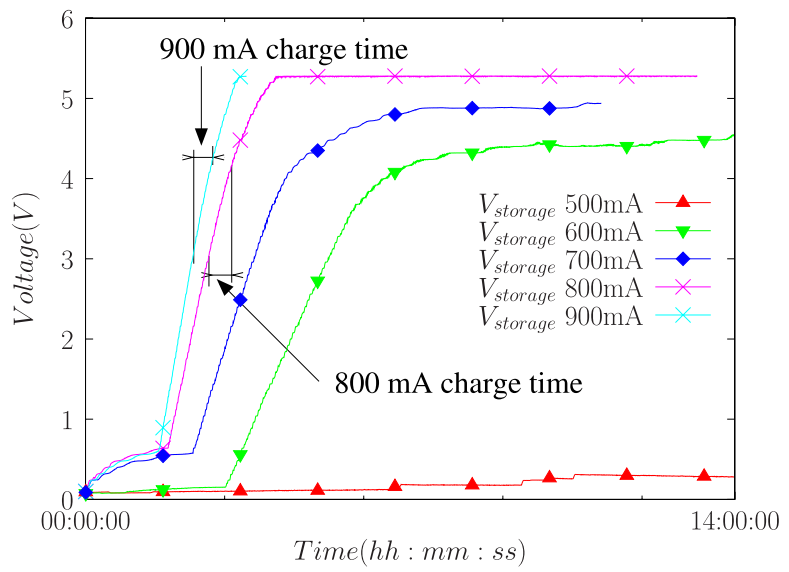

Fig. 15. Cellergy $120 \mathrm{mF} C_{\text {storage }}$ capacitor full charge for mains current from 500 to $900 \mathrm{~mA}$

with

$$
\left(\begin{array}{cc}
\sum_{n=1}^{N} t_{n}^{2} x & \sum_{n=1}^{N} t_{n} \\
\sum_{n=1}^{N} t_{n} & \sum_{n=1}^{N} 1
\end{array}\right)\left(\begin{array}{l}
M \\
B
\end{array}\right)=\left(\begin{array}{c}
\sum_{n=1}^{N} t_{n} \operatorname{Vst}_{\text {InstSlope }(n)} \\
\sum_{n=1}^{N} \operatorname{Vst}_{\text {InstSlope }(n)}
\end{array}\right) .
$$

Fig. 17 shows $V_{\text {storage }}$ readings, including also the computed

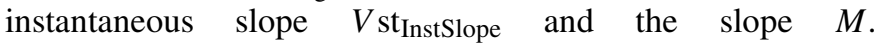
The $I_{\text {mains }}$ estimated is also shown, illustrating the close

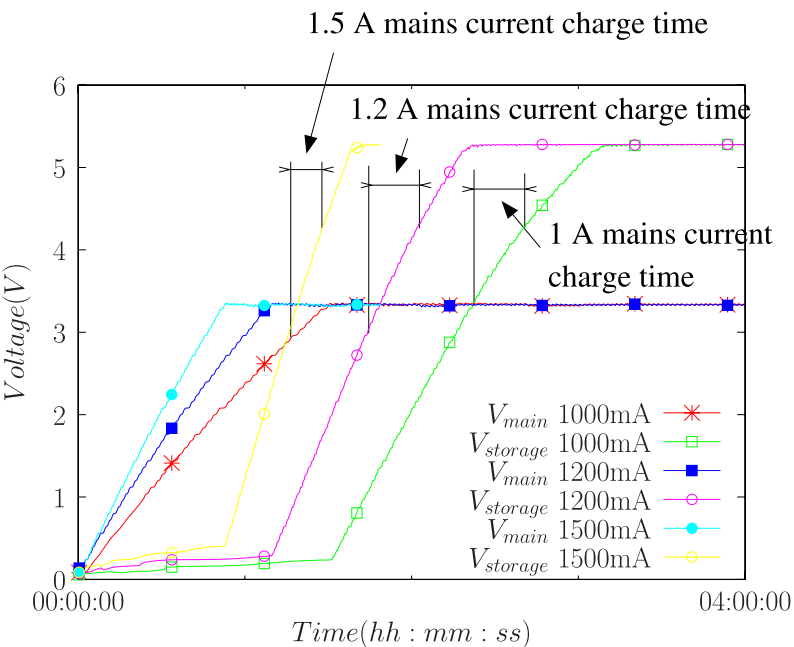

Fig. 16. Cellergy $120 \mathrm{mF} C_{\text {storage }}$ capacitor full charge for mains current from 1 to $1.5 \mathrm{~A}$.

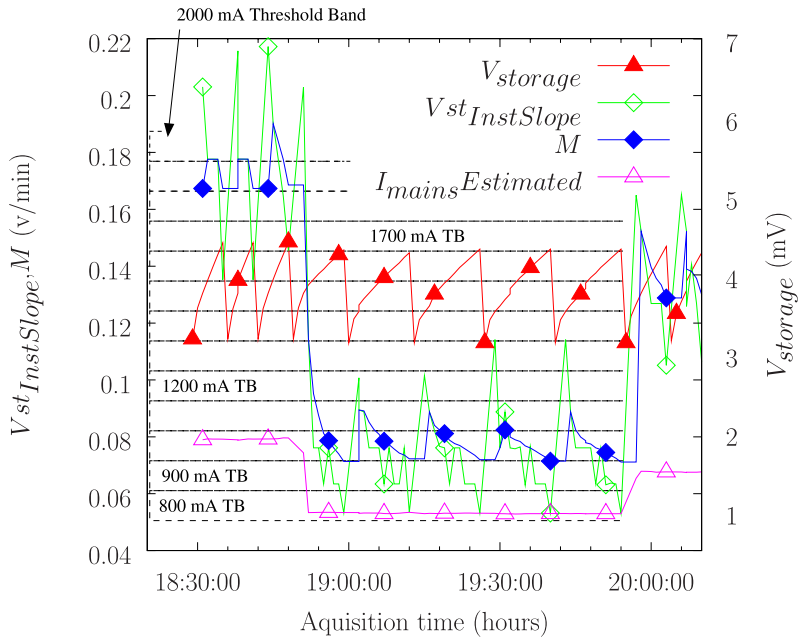

Fig. 17. $V_{\text {storage }}$ readings, including also computed instantaneous slope $V$ st InstSlope and the slope $M$ over a $2 \mathrm{~h}$ period.

relation between $M$ and the estimated current. Moreover, Fig. 17 presents a set of defined threshold bands (TB) such that for each band

$$
M_{\min _{I} \text { mains }}-D i<M<M_{\max _{I} \text { mains }}-D i
$$

where $M_{\min _{I} \text { mains }}$ and $M_{\max _{I} \text { mains }}$ are the minimum and maximum $M$ computed values for a given $I_{\text {mains }}, D$ is a subtracting term and $i$ is the $i$ th index of a reading within a discharge process. One TB is defined for each discrete estimated mains current implementing the lookup table shown in Table V. The TBs are an important part of the estimation process. Estimation is done by measuring $C_{\text {storage }}$ charge time. To this purpose, the device measures the $V_{\text {storage }}$ with a specified interval and sleeps the remaining time. The sleeping time is mandatory for any self-powered device and it has been set to $1 \mathrm{~min}$. The device must therefore operate in sleeping mode as long as possible thus entailing a small sample rate of $V_{\text {storage. The TBs tackle }}$ this inaccurate charge time measurements by defining bounds to the slope $M$ that is calculated based on the charge times. In this context, the quantization errors that occur when $V_{\text {storage }}$ 
TABLE V

$M$ TBS LOOKup TABle For OUR DeVICE

\begin{tabular}{ccccc}
\hline $\begin{array}{c}M_{\text {min }_{I} \text { mains }} \\
\text { V/min }\end{array}$ & & $\begin{array}{c}\text { Estimated current } \\
\mathrm{mA}\end{array}$ & & $\begin{array}{c}M_{\text {max }} \text { mains } \\
\text { V/min }\end{array}$ \\
\hline \hline- & $<$ & $700 \mathrm{~mA}$ & $<$ & 0.030 \\
\hline 0.030 & $<$ & $800 \mathrm{~mA}$ & $<$ & 0.058 \\
\hline 0.058 & $<$ & $900 \mathrm{~mA}$ & $<$ & 0.069 \\
\hline 0.069 & $<$ & $1000 \mathrm{~mA}$ & $<$ & 0.080 \\
\hline 0.080 & $<$ & $1100 \mathrm{~mA}$ & $<$ & 0.091 \\
\hline 0.091 & $<$ & $1200 \mathrm{~mA}$ & $<$ & 0.102 \\
\hline 0.102 & $<$ & $1300 \mathrm{~mA}$ & $<$ & 0.113 \\
\hline 0.113 & $<$ & $1400 \mathrm{~mA}$ & $<$ & 0.124 \\
\hline 0.124 & $<$ & $1500 \mathrm{~mA}$ & $<$ & 0.134 \\
\hline 0.134 & $<$ & $1600 \mathrm{~mA}$ & $<$ & 0.144 \\
\hline 0.144 & $<$ & $1700 \mathrm{~mA}$ & $<$ & 0.154 \\
\hline 0.154 & $<$ & $1800 \mathrm{~mA}$ & $<$ & 0.164 \\
\hline 0.164 & $<$ & $1900 \mathrm{~mA}$ & $<$ & 0.174 \\
\hline 0.174 & $<$ & $2000 \mathrm{~mA}$ & $<$ & 0.184 \\
\hline & & & &
\end{tabular}

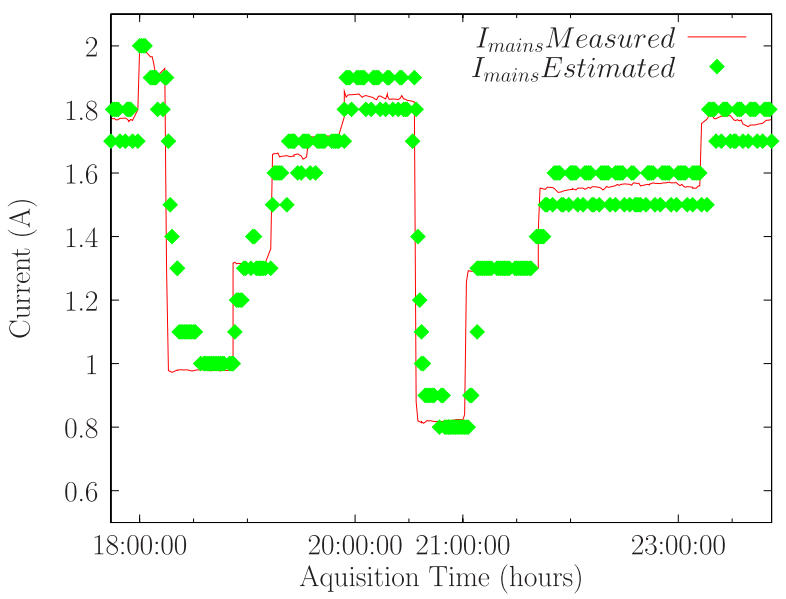

Fig. 18. System mains current estimations over a $6 \mathrm{~h}$ period.

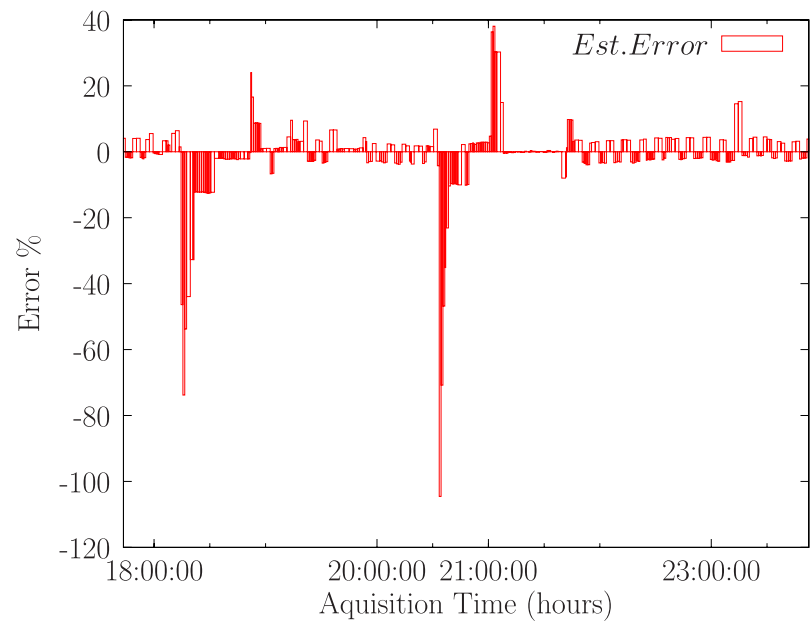

Fig. 19. System mains current estimations relative error over a $6 \mathrm{~h}$ period.

is measured by the MSP430 ADC have been considered negligible as they are also considered by the TBs. During one recharge process, the first computed $M$ value for one $I_{\text {mains }}$ is within its specific TB. The proposed estimation algorithm identifies the current mains average value if $M$ is computed within the respective $M_{\min _{I} \text { mains }}$ and $M_{\max _{I} \text { mains }}$ limits.
Parameter $D$ is introduced to cope with the fact that if $I_{\text {mains }}$ remains constant within a recharge process, smaller $M$ values are computed as time goes by. If $I_{\text {mains }}$ increases the computed $M$ value falls within the bounds of a higher TB. A smaller mains current is estimated if $M$ falls within the bounds of a lower TB. Fig. 18 shows mains current estimation obtained with this technique. $D$ is set to $D=0.0015$. Fig. 19 shows the estimation error obtained by the proposed device. The spikes that show large errors are introduced by the rapid mains current change that are sensed by the system only after the $1 \mathrm{~min}$ sleep time. The system behavior is determined by the small sample rate and its large capacitors. The system behaves therefore as a low-pass filter. Fast mains current variations are not directly sensed but accounted as an rms value.

\section{CONCLUSION}

Large buildings are responsible for a relevant part of the world's electrical energy consumption. Knowledge of energy consumption profiles is therefore important to determine saving policies and strategies. Even though technologies exist that are able to evaluate disaggregated energy consumption, they are expensive in material, installation, and maintenance. Due to the decentralized operation principle, WSN is an important tool to implement disaggregated electrical energy solutions. WSN presents however, large installation costs and frequently entail important operational difficulties, such as battery replacement. In this paper, a system that is able to estimate disaggregated current consumption has been presented. A single SCCT has been used to power a WSN node, which estimates at the same time the mains current flowing through a power line. Harvested energy is stored and accumulated so that a milliwatt device is powered, allowing WSN nodes operation. The proposed solution is able to sustain a WSN node communication using a complex communication protocol running on harvested energy. The proposed solution is built from mature devices and is therefore near market ready.

\section{ACKNOWLEDGMENT}

The authors would like to thank ISA-Intelligent Sensing Anywhere, S.A. (www.isa.pt), for their continuous support.

\section{REFERENCES}

[1] F. Salvadori et al., "Monitoring in industrial systems using wireless sensor network with dynamic power management," IEEE Trans. Instrum. Meas., vol. 58, no. 9, pp. 3104-3111, Sep. 2009.

[2] L. Atzori, A. Iera, and G. Morabito, "From 'smart objects' to 'social objects': The next evolutionary step of the internet of things," IEEE Commun. Mag., vol. 52, no. 1, pp. 97-105, Jan. 2014.

[3] A. Perttula, H. Leppakoski, M. Kirkko-Jaakkola, P. Davidson, J. Collin, and J. Takala, "Distributed indoor positioning system with inertial measurements and map matching," IEEE Trans. Instrum. Meas., vol. 63, no. 11, pp. 2682-2695, Nov. 2014.

[4] J. P. Amaro, F. J. T. E. Ferreira, R. Cortesao, N. Vinagre, and R. P. Bras, "Low cost wireless sensor network for in-field operation monitoring of induction motors," in Proc. IEEE Int. Conf. Ind. Technol. (ICIT), Mar. 2010, pp. 1044-1049.

[5] T. Taithongchai and E. Leelarasmee, "Adaptive electromagnetic energy harvesting circuit for wireless sensor application," in Proc. 6th Int. Conf. Elect. Eng./Electron., Comput., Telecommun., Inf. Technol. (ECTI-CON), vol. 1. May 2009, pp. 278-281. 
[6] A. Kosonen and J. Ahola, "Communication concept for sensors at an inverter-fed electric motor utilizing power-line communication and energy harvesting," IEEE Trans. Power Del., vol. 25, no. 4, pp. 2406-2413, Oct. 2010.

[7] M. Zhu, P. C. Baker, N. M. Roscoe, M. D. Judd, and J. Fitch, "Alternative power sources for autonomous sensors in high voltage plant," in Proc. IEEE Elect. Insul. Conf., May/Jun. 2009, pp. 36-40.

[8] R. H. Bhuiyan, R. A. Dougal, and M. Ali, "A miniature energy harvesting device for wireless sensors in electric power system," IEEE Sensors J., vol. 10, no. 7, pp. 1249-1258, Jul. 2010.

[9] N. M. Roscoe and M. D. Judd, "Harvesting energy from magnetic fields to power condition monitoring sensors," IEEE Sensors J., vol. 13, no. 6 , pp. 2263-2270, Jun. 2013.

[10] Z. Wu, Y. Wen, and P. Li, "A power supply of self-powered online monitoring systems for power cords," IEEE Trans. Energy Convers., vol. 28, no. 4, pp. 921-928, Dec. 2013.

[11] R. Moghe, A. Iyer, F. C. Lambert, and D. Divan, "A low-cost electricfield energy harvester for an MV/HV asset-monitoring smart-sensor," in Proc. IEEE Energy Convers. Congr. Expo. (ECCE), Sep. 2013, pp. 2676-2683.

[12] F. Guo, H. Hayat, and J. Wang, "Energy harvesting devices for high voltage transmission line monitoring," in Proc. IEEE Power Energy Soc. General Meeting, Jul. 2011, pp. 1-8.

[13] R. Shigeta et al., "Ambient RF energy harvesting sensor device with capacitor-leakage-aware duty cycle control," IEEE Sensors J., vol. 13, no. 8, pp. 2973-2983, Aug. 2013.

[14] J. Froehlich, E. Larson, S. Gupta, G. Cohn, M. S. Reynolds, and S. N. Patel, "Disaggregated end-use energy sensing for the smart grid," IEEE Pervasive Comput., vol. 10, no. 1, pp. 28-39, Jan./Mar. 2011.

[15] J. Gutierrez, J. F. Villa-Medina, A. Nieto-Garibay, and M. A. Porta-Gandara, "Automated irrigation system using a wireless sensor network and GPRS module," IEEE Trans. Instrum. Meas. vol. 63, no. 1, pp. 166-176, Jan. 2014

[16] R. Yan, H. Sun, and Y. Qian, "Energy-aware sensor node design with its application in wireless sensor networks," IEEE Trans. Instrum. Meas., vol. 62, no. 5, pp. 1183-1191, May 2013.

[17] Y. Kim, R. G. Evans, and W. M. Iversen, "Remote sensing and control of an irrigation system using a distributed wireless sensor network," IEEE Trans. Instrum. Meas., vol. 57, no. 7, pp. 1379-1387, Jul. 2008.

[18] J. P. Amaro, F. J. T. E. Ferreira, R. Cortesao, and J. Landeck, "Energy harvesting for ZigBee compliant wireless sensor network nodes," in Proc. 38th Аnnu. Conf. IEEE Ind. Electron. Soc. (IECON), Oct. 2012, pp. $2583-2588$.

[19] S. Baghaee et al., "Demonstration of energy-neutral operation on a WSN testbed using vibration energy harvesting," in Proc. 20th Eur. Wireless Conf., May 2014, pp. 1-6.

[20] J. Song and Y. K. Tan, "Energy consumption analysis of ZigBee-based energy harvesting wireless sensor networks," in Proc. IEEE Int. Conf. Commun. Syst. (ICCS), Nov. 2012, pp. 468-472.

[21] H. Kim, D. Choi, S. Gong, and K. Park, "Stray electric field energy harvesting technology using MEMS switch from insulated AC power line," Electron. Lett., vol. 50, no. 17, pp. 1236-1238, Aug. 2014.

[22] LTC3108 Datasheet, Linear Technology, Milpitas, CA, USA, Jul. 2014.

[23] CC2530 User Guide and Datasheet, Texas Instruments, Dallas, TX, USA, Jul. 2013.

[24] MSP430FR5739 User Guide and Datasheet, Texas Instruments, Dallas, TX, USA, Jul. 2013.

[25] V. Sedlakova, J. Sikula, J. Majzner, H. Navarova, M. Chvatal, and T. Zednicek, "Tantalum and niobium oxide capacitors: Field crystallization, leakage current kinetics and reliability," in Proc. 27th Int. Conf. Microelectron. (MIEL), May 2010, pp. 439-442.

[26] Aluminium Capacitors 150 CRZ Series, Vishay Intertechnology, Inc., Malvern, PA, USA, Apr. 2013.

[27] Cellergy Capacitors, Cellergy, Apr. 2013. [Online]. Available: http://www.cellergycap.com/

[28] Texas Instruments. Z-Stack-ZigBee Protocol Stack. [Online]. Available: http://www.ti.com/tool/z-stack, accessed Jul. 2013

[29] R. Severino, M. Alves, and A. Koubâa, "On the use of IEEE 802.15.4/ZigBee for time-sensitive wireless sensor network applications," M.S. thesis, ISEP, CISTER Research Center, Porto, Portugal, Oct. 2008

[30] Microchip Technology Inc. ZigBee Pro Stack. [Online]. Available: http://www.microchip.com, accessed Jun. 2012.

[31] E. Casilari, J. M. Cano-García, and G. Campos-Garrido, "Modeling of current consumption in 802.15.4/ZigBee sensor motes," Sensors, vol. 10, no. 6 , pp. $5443-5468,2010$.
[32] M.-S. Pan, C.-H. Tsai, and Y.-C. Tseng, "The orphan problem in ZigBee wireless networks," IEEE Trans. Mobile Comput., vol. 8, no. 11, pp. 1573-1584, Nov. 2009.

[33] J.-H. Lee, E.-S. Lee, and D.-S. Kim, "Network joining algorithm for mobile nodes in ubiquitous sensor networks," in Proc. 5th Int. Conf. Comput. Sci. Converg. Inf. Technol. (ICCIT), Nov./Dec. 2010, pp. 836-839.

[34] C. Hsiang, A.-W. Chen, C.-J. Chang, B.-Y. Shih, and C.-Y. Chen, "Development of mechanisms for MAC channel selection to improve the performance of IEEE 802.15.4 beacon-enabled network," in Proc. 3rd IEEE Int. Conf. Broadband Netw. Multimedia Technol. (IC-BNMT), Oct. 2010, pp. 598-602.

[35] J.-W. Kim, J. Kim, and D.-S. Eom, "Multi-dimensional channel management scheme to avoid beacon collision in LR-WPAN," IEEE Trans. Consum. Electron., vol. 54, no. 2, pp. 396-404, May 2008.

[36] Measuring Power Consumption With CC2430 and Z-Stack-Applcation Note AN053, Texas Instruments, Dallas, TX, USA, 2007.

[37] Z. Chen, C. Lin, H. Wen, and H. Yin, "An analytical model for evaluating IEEE 802.15.4 CSMA/CA protocol in low-rate wireless application," in Proc. 21st Int. Conf. Adv. Inf. Netw. Appl. Workshops (AINAW), vol. 2. May 2007, pp. 899-904.

[38] T. R. Park, T. H. Kim, J. Y. Choi, S. Choi, and W. H. Kwon, "Throughput and energy consumption analysis of IEEE 802.15.4 slotted CSMA/CA," Electron. Lett., vol. 41, no. 18, pp. 1017-1019, Sep. 2005.

[39] M. Mienkina, Filter-Based Algorithm for Metering Applications. Austin, TX, USA: Freescale Semiconductor, Inc., Nov. 2014.

[40] G. Hudson and T. Lean. (Sep. 2014). Open Energy Monitor. [Online]. Available: http://openenergymonitor.org/emon/

[41] J. Kim and C. S. Park, "A calibration technique for multibit stage pipelined A/D converters via least-squares method," IEEE Trans. Instrum. Meas., vol. 62, no. 12, pp. 3390-3392, Dec. 2013.

[42] M. D. Kusljevic, "On LS-based power frequency estimation algorithms," IEEE Trans. Instrum. Meas., vol. 62, no. 7, pp. 2020-2028, Jul. 2013.

[43] S. J. Miller, The Method of Least Squares. Providence, RI, USA: Brown Univ., 2010.

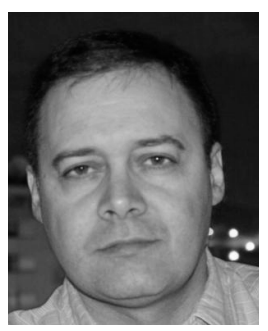

Jose Pedro Amaro received the Licentiate degree in electrical engineering from the University of Coimbra, Coimbra, Portugal, in 1992, and the M.Sc. degree in electronic systems from the Instituto Superior Técnico (IST), Technical University of Lisbon, Lisbon, Portugal, in 2004. He is currently pursuing the Ph.D. degree with the University of Coimbra.

He has been with the Nuclear Fusion Center, IST, and the Lisbon Cadence Center, Lisbon, where he worked on his M.Sc. thesis. He is currently an Assistant Professor with the Department of Electrical Engineering, Coimbra Institute of Engineering, Polytechnic Institute of Coimbra, Coimbra, and a Research Fellow with the Institute of Systems and Robotics, University of Coimbra. He is also involved in embedded systems development and fieldprogrammable gate array-based digital electronics, which are important topics that strongly relate to his academic and professional expertise. His current research interests include wireless sensor networks and energy harvesting solutions.

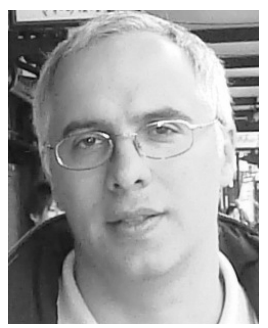

Rui Cortesão (M'05) received the B.Sc. degree in electrical engineering, the M.Sc. degree in systems and automation, and the Ph.D. degree in control and instrumentation from the University of Coimbra, Coimbra, Portugal, in 1994, 1997, and 2003, respectively.

He was a Visiting Researcher with the German Aerospace Center, Cologne, Germany, from 1998 to 2003, Stanford University, Stanford, CA, USA, in 2002, and the Laboratoire d'Informatique de Robotique et de Microélectronique de Montpellier-Centre National de la Recherche Scientifique, Paris, France, from 2004 to 2006, where he was involved in compliant motion control, data fusion, steer-by-wire, haptic manipulation, and surgical robotics. He has been a Researcher with the Institute of Systems and Robotics, since 1994, and an Assistant Professor with the Department of Electrical and Computer Engineering, University of Coimbra, where he teaches medical robotics and control. 


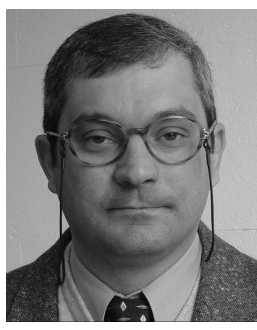

Jorge Landeck was born in 1967. He received the Ph.D. degree in technological physics from the University of Coimbra, Coimbra, Portugal.

He was a Professor with the Faculty of Science and Technology, University of Coimbra, and the Chief Technology Officer of Intelligent Sensing. He has over 20 years of experience in the areas of software, telemetry and distributed systems, and instrumentation. He has authored or co-authored several scientific publications in international refereed journals, managed several European projects, and developed several innovative remote management systems.

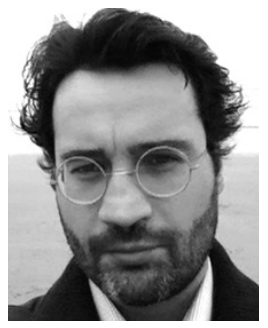

Fernando J. T. E. Ferreira (SM'09) received the Ph.D. degree in electrical engineering from the University of Coimbra, Coimbra, Portugal.

$\mathrm{He}$ has been a Researcher with the Institute of Systems and Robotics, University of Coimbra since 1998, where he is involved in motors and drives. He has participated in several European projects dealing with energy-efficient motor technologies. He is currently a Professor with the Department of Electrical Engineering, Polytechnic Institute of Coimbra, Coimbra. He has co-authored over 50 papers published in international journals and conference records, and presented at meetings.

Dr. Ferreira was a recipient of the Best Paper Award at the IEEE/IAS Industrial and Commercial Power Systems Technical Conference in 2001, and the Best Poster Presentation Award (for the technical competence displayed in the poster presentation) at the International Conference on Electrical Machines in 2010 . 